\title{
L. Derivative analysis; being a new and more comprehensive method of the transformation of functions than any hitherto discovered: extending not only to the extraction of the roots of equations, but also to the reduction of quantities from the multiples of powers or products to other equivalent expressions, by which the summation of any rational series may be readily effected
}

\section{Mr. Peter Nicholson}

To cite this article: Mr. Peter Nicholson (1823) L. Derivative analysis; being a new and more comprehensive method of the transformation of functions than any hitherto discovered: extending not only to the extraction of the roots of equations, but also to the reduction of quantities from the multiples of powers or products to other equivalent expressions, by which the summation of any rational series may be readily effected, Philosophical Magazine Series 1, 62:306, 244-252, DOI: 10.1080/14786442308644397

To link to this article: http://dx.doi.org/10.1080/14786442308644397 
state, was precipitated by carbonate of soda: $0.017 \mathrm{gr}$. of oxide of manganese was obtained, $=0.340$ per cent .

h.) The excess of hydrosulphuret of ammonia being removed, the liquid was decomposed by acetate of barytes; the sulphate of barytes was separated by filtration, and the fluid containing acetic acid being evaporated, and heated, and the residuum boiled in water, $0.217 \mathrm{gr}$. of carbonate of soda were obtained, containing 0.13057 of soda, $=2.611$ per cent. It must be observed, however, that in dissolving this carbonate of soda in water, some traces of magnesia remained undissolved, which had previously been dissolved in the alkaline solution.

$i$.) The residual matter $(h)$ was dissolved in muriatic acid, the barytes precipitated by sulphuric acid, and separated on a filter; and the liquid in a state of ebullition precipitated by carbonate of soda $: 0.271 \mathrm{gr} .=5.420$ per cent. of pure magnesia was obtained, which entirely crystallized with sulphuric acid, into sulphate of magnesia.

The fluoric and phosphoric acids were sought for, in an assay made for the purpose, but no traces of them were discovered. Erlan, therefore, consists of

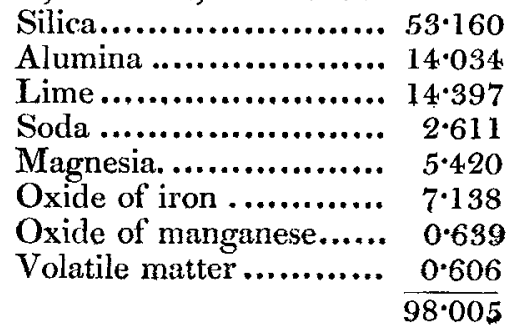

L. Derivative Analysis; being a new and more comprehensive Method of the Transformation of Functions than any hitherto discovered: extending not only to the Extraction of the Roots of Equations, but also to the Reduction of Quantities from the Multiples of Porvers or Products to other equivalent Expressions, by which the Summation of any rational Series may be readily effected. By Mr. Peter Nrcholson*. 5 Claremont-place, Judd-street.

To the Editors of the Philosophical Magazine and Journal.

$$
\text { Part 1.-Multiplication. }
$$

MULTIPLICATION is performed in the usual way; but instead of the compound coefficients of the entire product, substitute a letter for the amount or aggregate of each such coefficient; then as many equations as the entire product

* Communicated by the Author. 
has coefficients will be formed, which will show the relation between the succeeding and the preceding coefficients of the entire product, or between the coefficients of the entire product and those of the multiplicand and multiplier.

Ex. 1. Multiply $\mathrm{A}+\mathrm{B} x+\mathrm{C} x^{2}+\mathrm{D} x^{3}+\mathrm{E} x^{4}+\& x$. by the binomial $a+x$.

$$
\begin{aligned}
& \text { Operation. } \\
& \mathrm{A}+\mathrm{B} x+\mathrm{C} x^{2}+\mathrm{D} x^{3}+\mathrm{E} x^{4}+\& \mathrm{c} . \\
& \frac{a+x}{a \mathrm{~A}+a \mathrm{~B} x+a \mathrm{C} x^{2}+a \mathrm{D} x^{3}+a \mathrm{E} x^{4}+8 \mathrm{c} .} \\
& \frac{\mathrm{A} x+\mathrm{B} x^{2}+\mathrm{C} x^{3}+\mathrm{D} x^{4}+8 \mathrm{c}}{\mathrm{A}_{\mathrm{t}}+\mathrm{B}_{\mathrm{t}} x+\mathrm{C}_{1} x^{2}+\mathrm{D}_{\mathrm{r}} x^{3}+\mathrm{E}_{1} x^{4}+\& \mathrm{c} .}
\end{aligned}
$$

From which we have the following derivative equations, viz.

$$
\begin{aligned}
& \mathrm{A}_{\mathrm{r}}=\quad a \mathrm{~A} \\
& \mathrm{~B}_{1}=\mathrm{A}+a \mathrm{~B} \\
& \mathrm{C}_{\mathrm{t}}=\mathrm{B}+a \mathrm{C} \\
& \mathrm{D}_{\mathrm{r}}=\mathrm{C}+a \mathrm{D}
\end{aligned}
$$

Hence it appears that the entire product may be derived from the multiplicand, and the constant part of the multiplier. Since any coefficient of the entire product is equal to the partial product of the corresponding coefficient of the multiplicand, and the constant part of the multiplier plus the preceding coefficient of the multiplicand.

Ex. 2. Multiply $x^{n-1}+\mathrm{B} x^{n-2}+\mathrm{C} x^{n-3}+\mathrm{D} x^{n-4}+\mathrm{E} x^{n-5}+8 x$. by the binomial $x+a$.

$$
\begin{aligned}
& x^{n-1}+\mathrm{B} x^{n-2}+\mathrm{C} x^{n-s}+\mathrm{D} x^{n-4}+\mathrm{E} x^{n-5}+\& \mathrm{c} . \\
& \frac{x+a}{x^{n}+\mathrm{B} x^{n-1}+\mathrm{C} x^{n-2}+\mathrm{D} x^{n-3}+\mathrm{E} x^{n-4}+8 \mathrm{c} .} \\
& \frac{+a x^{n-1}+a \mathrm{~B} x^{n-2}+a \mathrm{C} x^{n-3}+a \mathrm{D} x^{n-4}+\& c .}{x_{n}+\mathrm{B}_{1} x^{n-1}+\mathrm{C}_{\mathbf{r}} x^{n-2}+\mathrm{D}_{1} x^{n-3}+\mathbf{E}_{1} x^{n-4}+\& \mathrm{c} .}
\end{aligned}
$$

Whence we have the following derivative equations, viz.

$$
\begin{aligned}
& \mathrm{B}_{\mathrm{1}}=\mathrm{B}+a \\
& \mathrm{C}_{\mathrm{t}}=\mathrm{C}+a \mathrm{~B} \\
& \mathrm{D}_{1}=\mathrm{D}+a \mathrm{C} \\
& \mathrm{E}_{1}=\mathrm{E}+a \mathrm{D} \\
& 8 \mathrm{c} .
\end{aligned}
$$

From which it appears that the entire product may be derived from the multiplicand; for the coefficient of any term of the entire product is equal to the coefficient of the corresponding term of the multiplicand plus the partial product of the preceding term of the multiplicand, and the second part of the multiplier.

Ex. 3. Multiply the series $1+a x+a^{2} x^{2}+a^{3} x^{3}+\& c$. by the series $1+b x+b^{2} x^{2}+b^{3} x^{3}+8 c$.

Put 
Put $\mathrm{B}=a, \mathrm{C}=a^{2}, \mathrm{D}=a^{3}$ \&x. and the operation will be

$$
\begin{array}{r}
1+\mathrm{B} x+\mathrm{C} x^{2}+\mathrm{D} x^{3}+\& c . \\
1+b x+b^{2} x^{2}+b^{3} x^{3}+8 \mathrm{c} . \\
\hline 1+\mathrm{B} x+\mathrm{C} x^{2}+\mathrm{D} x^{3}+8 \mathrm{c} . \\
b x+b \mathrm{~B} x^{2}+b \mathrm{C} x^{4}+8 \mathrm{c} . \\
+b^{2} x^{2}+b^{2} \mathrm{~B} x^{2}+\& \mathrm{c} . \\
+b^{3} x^{3}+\& \mathrm{c} . \\
+8 \mathrm{c} .
\end{array}
$$

Where $\mathrm{B}_{1}=\mathrm{B}+\mathrm{b}$,

$$
\begin{aligned}
& \mathbf{C}_{1}=\mathbf{C}+b \mathbf{B}+b^{2}=\mathbf{C}+b(\mathbf{B}+b)=\mathbf{C}+b \mathbf{B}_{\mathrm{r}} \\
& \mathbf{D}_{\mathbf{1}}=\mathbf{D}+b \mathbf{C}+b^{2} \mathbf{B}+b^{3}=\underset{\mathrm{D}+b}{\delta c}\left(\mathbf{C}+b \mathbf{B}+b^{2}\right)=\mathrm{D}+b \mathbf{C}_{\mathbf{1}} \\
& \text { sc. }
\end{aligned}
$$

In the same manner by taking the series $1+\mathrm{B}_{1} x^{2}+\mathrm{C}_{1} x^{2}+$ $\mathbf{D}_{1} x^{3}+\& c$. as a multiplicand and the series $1+c x+c^{2} x^{2}+c^{3} x^{3}+$ \&c. as a multiplier; then if the entire product be $1+\mathrm{B}_{2} x+$ $\mathbf{C}_{2} x^{2}+\mathbf{D}_{2} x^{3}+\& c$. we shall have by the same law

$$
\begin{aligned}
& \mathrm{B}_{2}=\mathrm{B}_{1}+\mathrm{C} \\
& \mathrm{C}_{2}=\mathrm{C}_{1}+c \mathrm{~B}_{1}+c^{2}=\mathrm{C}_{2}+c \mathrm{~B}_{2} \\
& \mathrm{D}_{2}=\mathrm{D}_{1}+c \mathrm{C}_{1}+c^{2} \mathrm{~B}_{1}+c^{3}=\mathrm{D}_{1}+c\left(\mathrm{C}_{1}+c \mathrm{~B}_{1}+c^{2}\right)=\mathrm{D}_{1}+c \mathrm{C}_{2} \\
& \& \text { sc. }
\end{aligned}
$$

and so on for the product of any number of series; therefore, arranging these values according to the number of products, there will arise $\mathrm{B}_{1}=\mathrm{B}+b\left|\mathrm{C}_{1}=\mathrm{C}+b \mathrm{~B}_{1}\right| \mathrm{D}_{1}=\mathrm{D}+b \mathrm{C}_{1} \mid$

$$
\begin{array}{c|c|c}
\mathbf{B}_{2}=\mathrm{B}_{1}+c & \mathrm{C}_{2}=\mathrm{C}_{1}+c \mathrm{~B}_{2} & \mathrm{D}_{2}=\mathrm{D}_{1}+c \mathbf{C}_{2} \\
\mathbf{B}_{3}=\mathrm{B}_{2}+d & \mathbf{C}_{3}=\mathrm{C}_{2}+d \mathrm{~B}_{3} & \mathrm{D}_{3}=\mathrm{D}_{2}+d \mathbf{C}_{j} \\
\& c . & \& c . & \& c .
\end{array}
$$

Let it be required to find all the combinations of the letters $a, b, c$, equally with one another to the third order.

Now observing that $\mathrm{B}=\alpha, \mathrm{C}=\alpha^{2}, \mathrm{D}=\alpha^{3}$, then will

$$
\mathrm{B}_{1}=a+b
$$

1st order. $\mathbf{B}_{2}=-+$

$$
\mathrm{C}_{1}=a^{2}+a b+b^{2}
$$

$2 \mathrm{~d}$ order. $\mathrm{C}_{\mathbf{2}}=-$

$$
\mathrm{D}_{1}=a^{3}+a^{2} b+a b^{2}+b^{3}
$$

$3 \mathrm{~d}$ order. $\mathrm{D}_{2}=-a^{2} c+a b c+b^{2} c+a c^{2}+b c^{2}+c^{3}$

$8 \mathrm{c}$.

Where the long line stands for all the combinations of the next line above it.

Again, let it be required to find all the orders of the combinations of the letters $a a a, b b, c$, or $a^{3}, b^{2}, c$, or let all the divisors of 360 be required; now $360=2^{3} \cdot 3^{2} .5=a^{3} b^{2} c$.

Here $B_{1}=a+b$

1 st order. $\mathrm{B}_{2}=-+c$

$$
\overline{\mathrm{C}_{1}}=\overline{a^{2}+a} b \overline{b^{2}}
$$

$2 \mathrm{~d}$ order. $\mathrm{C}==-1+a c+b$ for $c^{2}$ is not wanted 
$\mathrm{D}_{1}=a^{3}+a^{2} b+a b^{2}$ for $b^{3}$ is not wanted

3d order. $\mathrm{D}_{2}=-\frac{}{2}+a^{2} c+a b c+b^{2} c$

$\mathbf{E}_{1}=a^{3} b+a^{2} b^{2}$ no higher than $a^{3}$ or $b^{2}$ being wanted

4th order. $\mathrm{E}_{2}=-+a^{3} b c+a^{2} b^{2} c$

$\mathrm{F}_{\mathrm{x}}=a^{3} b^{2}$

5th order. $\mathrm{F}_{2}=-+a^{3} b c+a^{2} b^{2} c$

$\mathrm{G}_{1}=0$

6th order. $\mathrm{G}_{2}=a^{3} b^{2} c$

\section{Part II.-Division.}

Division is performed in the usual way, viz. by arranging the parts of the dividend in a line according to the natural or inverse order of the powers of the variable, and the parts of the divisor in the same order.

Divide the first part of the dividend by the first part of the divisor, and the result is the first part of the quotient.

Multiply the first part of the quotient successively, by every part of the divisor, and place the products so that the powers of the variable may be under the same powers in the dividend.

Draw a line underneath and write in a line below the line thus drawn the same powers of the variable as those immediately above, except in the first place, and prefix a new letter to each power as a coefficient which will form the first remainder.

Annex the next part of the dividend from which no subtraction has been made to this remainder, and consider this remainder so increased as a second dividend; then proceed to find the second or next part of the quotient, and the third or next dividend as before; and so on, as far as may be necessary. In any convenient place write the letters thus substituted, and their values, in the form of equations; that is, every letter equal to the aggregate of the two coefficients of the corresponding power above, considering the sign of the lower of these two changed by subtraction.

Then the table thus formed will show the law of derivation by which the real quotient may be obtained.

Ex. 1. Divide $\alpha+\beta x+\gamma x^{2}+\delta x^{3}+\delta c$. by $1-b x$ put $A=\alpha$; then proceed with the operation,

Dividend.

$$
\begin{aligned}
& \mathrm{A}+\beta x+\gamma x^{2}+\delta x^{3}+\delta \mathrm{c} \text {. } \\
& \mathrm{A}-\mathbf{\Lambda} b x \\
& \mathrm{~B} x+\gamma x^{2} \\
& \mathrm{~B} x-\mathrm{B} b x^{2} \\
& \mathrm{C} x^{2}+0 x^{3} \\
& \mathrm{C} x^{3}-\mathrm{C} b x^{3} \\
& \mathrm{D} r^{3}+8 \mathrm{c} \text {. } \\
& \text { ic. } \\
& 1-b x \\
& \begin{array}{l}
\text { quotient } \\
\mathrm{A}+\mathrm{B} x+\mathrm{C} x^{2}+\mathrm{Sc} .
\end{array}
\end{aligned}
$$

Divisor. 

viz.

By this operation we have the following derivative table,

$$
\begin{gathered}
\mathrm{A}=\alpha \\
\mathrm{B}=\mathrm{A} b+\beta \\
\mathrm{C}=\mathrm{B} b+\gamma \\
\mathrm{D}=\mathrm{C} b+\delta \\
\text { \&c. }
\end{gathered}
$$

From which it appears that the $n$th coefficient of the quotient is equal to the product of the next preceding coefficient, and the coefficient of the second term of the divisor plus the $n$th coefficient of the dividend. Whence by the table we derive the coefficients of the quotient thus,

$$
\begin{aligned}
\mathbf{A}=\quad \alpha=\alpha & =\alpha \\
\mathbf{B}=\mathrm{A} b+\beta=\alpha b+\beta & \mathbf{C}=\mathbf{B} b+\gamma=\alpha b^{2}+\beta b+\gamma \\
\mathbf{D}=\mathbf{C} b+\delta=\alpha b^{3}+\beta b^{2}+\gamma b+\delta & \quad \& \mathbf{c} . \quad 8 .
\end{aligned}
$$

Whence

$$
\mathrm{A}+\mathrm{B} x+\mathrm{C} x^{2}+\& \mathrm{c} .=\alpha+(\alpha b+\beta) x+\left(\alpha b^{2}+\beta b+\gamma\right) x^{2}+8 \mathrm{c} .
$$

$E x$. 2. Divide the infinite series $\alpha+\beta x+\gamma x^{2}+\delta x^{3}+\varepsilon x^{4}+\& c$. by $a-b x-c x^{2}$.

$$
\begin{aligned}
& \text { Operation. } \\
& \text { Dividend. Divisor. } \\
& \alpha+\beta x+\gamma x^{2}+\delta x^{3}+\varepsilon x^{4}+\& c . \quad \mid a-b x-c x^{2} \\
& \begin{array}{l}
\alpha-\frac{b \alpha}{a} x-\frac{c a}{a} x^{2} \\
\mathrm{~B} x+\mathrm{B}_{\mathrm{t}} x^{2}+\delta x^{3} \\
\mathrm{~B} x-\frac{\frac{b \mathrm{~B}}{a} x^{2}-\frac{c \mathrm{~B}}{a} x^{3}}{\frac{\mathrm{C} x^{3}+\mathrm{C}_{\mathrm{t}} x^{3}+\varepsilon x^{4}}{\mathrm{C}}}
\end{array} \\
& \frac{\mathrm{C} x^{2}-\frac{b \mathrm{C}}{a} x^{3}-\frac{c \mathrm{C}}{a} x^{4} \& c .}{\mathrm{D} x^{3}+\mathrm{D}_{1} x^{4}+\& \mathrm{c} .}
\end{aligned}
$$

From which operation we have the following derivative table, viz.

$$
\begin{aligned}
& \mathbf{B}=\frac{\alpha b+\beta a}{a} \\
& \mathrm{~B}_{1}=\frac{a c+\gamma a}{a}\left|\begin{array}{l}
\mathrm{C}=\frac{\mathrm{B} b+\mathrm{B}_{\mathrm{r}} a}{a} \\
\mathrm{C}_{\mathbf{1}}=\frac{\mathrm{B} c+\delta a}{a}
\end{array}\right| \mathrm{D}=\left.\frac{\mathrm{C} b+\mathrm{C}_{\mathbf{1}} a}{a}\right|_{\text {\&c. }} ^{\text {\&c. }}
\end{aligned}
$$

From this table we derive the real quotient

$$
\frac{\alpha}{a}+\frac{\alpha b+\beta a}{a^{2}} x+\frac{a b^{2}+\beta a b+\alpha a c+\gamma a^{2}}{a^{3}} x^{2}+\& c .
$$


But if the first part $a$ of the divisor were unity, the derivative table would be simply

$$
\begin{aligned}
& \mathrm{B}=\alpha b+\beta \\
& \mathrm{B}_{1}=\alpha c+\gamma
\end{aligned}\left|\begin{array}{l}
\mathrm{C}=\mathrm{B} b+\mathrm{B}_{1} \\
\mathrm{C}_{1}=\mathrm{B} c+\delta
\end{array}\right| \begin{aligned}
& \mathrm{D}=\mathrm{C} b+\mathrm{C}_{1} \\
& \mathrm{D}_{1}=\mathrm{C} c+\varepsilon^{\prime}
\end{aligned}\left|\begin{array}{c}
\mathrm{E}=\underset{\mathrm{D} b}{\& \mathrm{c} .}
\end{array}\right|_{\& \mathrm{c} .}
$$

And the quotient derived from this table would be simply

$$
\begin{gathered}
\alpha+\mathbf{B} x+\mathbf{C} x^{2}+\& c= \\
\alpha+(\alpha b+\beta) x+\left(\alpha b^{2}+\beta b+\alpha c+\gamma\right) x^{2}+\alpha c .
\end{gathered}
$$

Ex. 3. Divide the series $\alpha+\beta x+\gamma x^{2}+\delta x^{3}+\varepsilon x^{4}+\& c$. by the series $1-b x-c x^{2}-d x^{3}-e x^{4}-8 c$.

\section{Operation.}

\section{Dividend.}

Divisor.

$\alpha+\beta x+\gamma x^{2}+\delta x^{3}+\varepsilon x^{4}+\varepsilon c$. Quotient $\alpha-\alpha b x-\alpha c x^{2}-\alpha d x^{3}-\alpha e x^{4}-\& \mathrm{c} . \mid \alpha+\mathrm{B} x+\mathrm{C} x^{2}+\mathrm{D} x^{3}+\& \mathrm{c}$.

$\mathrm{B} x+\mathrm{B} x^{2}+\mathrm{B} x^{3}+\mathrm{B}_{3} x^{4}+8 \mathrm{c}$. $\frac{\mathrm{B} x-b \mathrm{~B} x^{2}-c \mathrm{~B} x^{3}-d \mathrm{~B} x^{4}-8 \mathrm{c} \text {. }}{\mathrm{C} x^{2}+\mathrm{C} x^{3}+\mathrm{C} x^{4}+\& \mathrm{c} \text {. }}$ $\mathrm{C} x^{2}-b \mathrm{C} x^{3}-c \overline{\mathrm{C}} x^{4}-8 \mathrm{c}$. $\mathrm{D} x^{3}+\mathrm{D} x^{4}+8 \mathrm{c}$. $\mathrm{D} x^{3}+6 \mathrm{D} x^{4}-8 \mathrm{c}$.

$\mathrm{E} x^{7}+8 \mathrm{c}$.

$\mathrm{E} x^{4}-8 \mathrm{c}$.

$\mathrm{By}$ this operation we have the following derivative equations, viz.

$$
\begin{aligned}
& \mathrm{B}=b \alpha+\beta \\
& B_{1}=c \alpha+\gamma \\
& \mathrm{B}_{2}=d \alpha+\delta \\
& \mathrm{B}_{5}=e \alpha+8 \\
& \mathrm{C}=b \mathrm{~B}+\mathrm{B}_{\mathrm{i}} \\
& \mathrm{C}_{1}=c \mathrm{~B}+\mathrm{B}_{2} \\
& \mathrm{C}=d \mathrm{~B}+\mathrm{B}_{3} \\
& \text { \&c. } \\
& \text { \&c. } \\
& \begin{array}{c}
\mathrm{D}=b \mathrm{C}+\mathrm{C}_{1} \\
\mathrm{D}_{1}=c \mathrm{C}+\mathrm{C}_{2} \\
\text { \&c. }
\end{array} \\
& \left|\begin{array}{c}
\mathrm{E}=b \mathrm{D}+\mathrm{D}_{1} \\
\& \mathrm{c} .
\end{array}\right| \& \mathrm{c} .
\end{aligned}
$$

By multiplying and adding as this table directs, we shall have the real coefficients of the powers of $x$ in the quotient, viz.

$$
\begin{aligned}
& \mathrm{A}=\alpha \\
& \mathbf{B}=\alpha b+\beta \\
& \mathbf{C}=\alpha b^{2}+\beta b+\alpha c+\gamma \\
& \mathrm{D}=\alpha b^{3}+\beta b^{2}+\alpha c b+\gamma b+\alpha b c+\beta c+\alpha d+\delta \\
& \quad \text { \&c. }
\end{aligned}
$$

Or, if the divisor had been $a-b x-c x^{2}-d x^{3}-\& c$. instead of $1-b x-c x^{2}-d x^{3}-8 c$. and if $A$ had been the first term of

Vol. 62. No. 306. Oit. 1823. 
the quotient, B, C, D, \&c. the coefficients of the following terms, we should have had

$$
\begin{aligned}
& \mathrm{A}=\frac{a}{a} \\
& \mathrm{~B}=\frac{a b+\beta a}{a^{2}} \\
& \mathrm{C}=\frac{\alpha b^{2}+\beta a b+\alpha a c+\gamma a^{2}}{a^{3}} \\
& \mathrm{D}=\frac{a b^{3}+\beta a b^{2}+? a u b c+\gamma a^{2} b+\beta a^{2} c+\alpha a^{2} d+\delta \alpha^{3}}{a^{4}} \\
& \& c . \quad \& \mathrm{c} .
\end{aligned}
$$

That is, by making up the sum of the indices to the same number as the highest in each part of each numerator, and making the denominators respectively $a, a^{2}, a^{3}, a^{4}$, \&c., so that if we have the coefficients in one way, we can easily find them in the other.

But if $a, \beta, \gamma, \& c$, and $a, b, c, \& c$. had been given in numbers, the values of $\mathrm{A}, \mathrm{B}, \mathrm{C}$, the coefficients of the quotient, would have been found much more easily by the rule directed in the table, as we shall have occasion to show hereafter.

Ex. 4. Divide the series $a x^{m}+\beta x^{m-1}+\gamma x^{m-2}+\delta x^{m-3}+\& c . b y$ the series $x^{n}-b x^{n-1}-c x^{n-2}-d x^{n-3}-\& c$.

\section{Divisor.}

$\alpha x^{m}+\beta x^{m-1}+\gamma x^{m-2}+\delta x^{m-3}+8 \mathrm{c} \cdot$ Quotient.

$\frac{\alpha x^{m}-b \alpha x^{m-1}-c a x^{m-2}-d \alpha x^{2-3}-8 \mathrm{c} \cdot \alpha_{\alpha} x^{m-n}+\mathrm{B} x^{m \cdot n-1}+\mathrm{C} x^{m-n-2}+\& \mathrm{c} .}{\mathbf{B} x^{m-1}+\mathrm{B}_{1} x^{m-2}+\mathrm{B}_{2} x^{m-3}+\& \mathrm{c} .}$

$$
\begin{array}{r}
\frac{\mathrm{B} x^{m-1}-b \mathrm{~B} x^{m-2}-c \mathrm{~B} x^{m-3}-8 \mathrm{c} .}{\mathrm{C} x^{m-2}+\mathbf{C} x^{m-3}+8 \mathrm{c} .} \\
\mathbf{C} x^{m-2}-b \mathbf{C} x^{m-3}-8 \mathrm{c} . \\
\frac{\mathbf{D} x^{m-3}+8 \mathrm{c} .}{\mathrm{D} x^{m-3}-8 \mathrm{c} .}
\end{array}
$$

By this operation we have the following derivative equations,

$$
\begin{aligned}
& \mathrm{B}=b \alpha+\beta \\
& \mathrm{B}_{\mathrm{L}}=c \alpha+\gamma \quad \mathbf{C}=b \mathbf{B}+\mathrm{B}_{\mathrm{r}}
\end{aligned}
$$

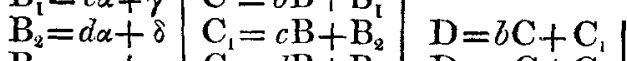

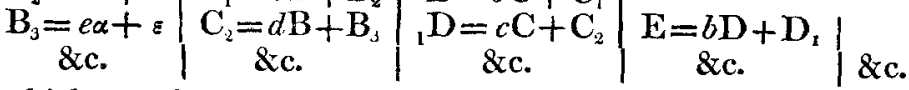

which are the same as those in the table of the preceding example. 
Mr. P. Nicholson on derivative Analysis.

$E x .5$. Divide the quantity $\mathbf{A}$ by the binomial $v-e$.

Divisor.

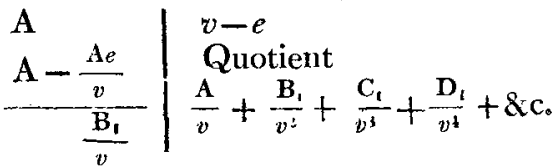

$$
\begin{aligned}
& \frac{\frac{\mathbf{B}_{1}}{v}-\frac{e \mathbf{B}_{\mathrm{t}}}{v^{2}}}{\frac{\mathbf{C}_{4}}{v^{3}}} \\
& \frac{\mathrm{C}_{1}}{v^{2}}-\frac{e \mathrm{C}_{\mathrm{t}}}{v^{3}} \\
& \frac{D_{1}}{v^{3}}-8 \mathrm{c} \text {. }
\end{aligned}
$$

By this operation we have the following derivative equations, viz.

$$
\begin{gathered}
\mathrm{B}_{1}=e \mathbf{A} \\
\mathrm{C}_{1}=e \mathbf{B}_{1} \\
\mathrm{D}_{1}=e \mathrm{C}_{1} \\
\& \mathrm{c} .
\end{gathered}
$$

Hence it appears that any quotient figure is equal to the product of the next preceding quotient figure by the second part of the divisor.

$E x$. 6. Divide the series $\mathrm{B}+\frac{\mathrm{A}}{v}+\frac{\mathrm{B}_{1}}{v^{2}}+\frac{\mathrm{C}_{1}}{v^{3}}+\frac{\mathrm{D}_{1}}{v^{t}}+\& \mathrm{c}$. (which is the quotient in the preceding example increased by the quantity $\mathrm{B}$ ) by the binomial $v-e$ (which is the same divisor as in the preceding example.

$$
\begin{aligned}
& \begin{array}{l|l}
\mathbf{B}+\frac{\mathbf{A}}{v}+\frac{\mathbf{B}_{1}}{v^{2}}+\frac{\mathbf{C}_{1}}{v^{3}}+\frac{\mathbf{D}_{1}}{v^{4}}+\& \mathrm{c} . & \begin{array}{l}
v-e \\
\text { Quotient } \\
\frac{\mathbf{B}}{v}+\frac{\mathbf{B}_{2}}{v^{2}}+\frac{\mathbf{C}_{2}}{v^{3}}+\frac{\mathbf{D}_{2}}{v^{4}}+\& \mathrm{c} .
\end{array} \\
\mathbf{B}-\frac{e \mathbf{B}}{v} & .
\end{array} \\
& \frac{\mathbf{B}_{2}}{\eta}+\frac{\mathbf{B}_{\mathbf{i}}}{v^{2}} \\
& \frac{\mathrm{B}_{2}}{v}+\frac{e \mathrm{~B}_{1}}{v^{2}} \\
& \overline{\mathrm{C}_{2}}+\frac{\mathrm{C}_{t}}{v^{3}} \\
& \frac{\mathrm{C}_{2}}{v^{2}}-\frac{c \mathbf{C}_{2}}{v^{3}} \\
& \overline{\frac{\mathrm{D}_{2}}{2^{3}}+\frac{\mathrm{D}_{1}}{v^{2}}}
\end{aligned}
$$


By this operation we have the following derivative equations,

$$
\begin{gathered}
\mathbf{B}_{2}=e \mathrm{~B}+\mathbf{A} \\
\mathbf{C}_{2}=e \mathbf{B}_{2}+\mathbf{B}_{s} \\
\mathrm{D}_{2}=e \mathbf{C}_{2}+\mathbf{C}_{1} \\
\varepsilon \mathrm{c} .
\end{gathered}
$$

which show the law of derivation.

['To be continued.]

LI. Discovery of the secret Destroyers of the Trees in St.James's Park.

To the Editors of the Philosophical Magazine and Journal.

$7 \mathrm{TE}$ alterations which are now taking place in the Parks, and particularly in St. James's Park, evidently excite a good deal of public interest. Few persons, however, seem aware that the greatest change which is about to be effected in the appearance of these "Lungs of London," is one which is contemplated by none with less pleasure than by those who have the care of them. Few persons suspect, for instance, that in a very short period St. James's Park will be clothed in the dapper dress of a nursery plantation, and will have lost those shady avenues and that antiquated appearance which are all associated with the recollections of times gone past. So rapidly, however, is it advancing to this state, that every person who is in the habit of entering it must perceive that, unless some remedy be quickly applied, a few months have only to elapse when there will be scarcely any thing green in it but the grass. Of the saplings which have been lately planted I do not speak; but it is manifest that every thing: deserving the name of a tree, a few limes only excepted, is rapidly disappearing. In spring we see the leaves sprout forth from the venerable trunks in all the luxuriance of vegetation, when of a sudden they are blasted as if by lightning, the bark falls from the stem, and long ere winter the finest tree perhaps in the park is only fit for fire-wood. Whole rows have thus disappeared and are still rapidly disappearing in the Mall and Bird-Cage Walk; and as it is anticipated that the public will esteem this open condition of the park to be little conducive to its beauty, even if it should add to its salubrity, great pains have of course been taken to find out the cause of the mischief.

As it was clear that the trees died in consequence of being completely stripped of their bark, rewards were at first offered for the discovery of the persons who so mischievonsly barked them ; but in vain. It was observed, however, very ingeniously, 